\title{
UN SISTEMA PARA LA DETECCION DE ANTIOXIDANTES VOLATILES COMUNMENTE EMITIDOS DESDE ESPECIAS Y HIERBAS MEDICINALES
}

\author{
Edgar Pastene*, Maritza Gómez y Hernán Speisky \\ Laboratorio de Antioxidantes, Instituto de Nutrición y Tecnología de los Alimentos, Universidad de Chile, Macul 5540, Macul - \\ Santiago, Chile \\ Luís Núñez-Vergara \\ Departamento de Química Farmacológica y Toxicológica, Facultad de Ciencias Químicas y Farmacéuticas, Universidad de Chile, \\ Olivos 1007, Independencia, Santiago - Chile
}

Recebido em 16/1/08; aceito em 29/8/08; publicado na web em 26/1/09

\begin{abstract}
A SYSTEM FOR DETECTION OF VOLATILE ANTIOXIDANT COMMONLY EMITTED FROM SPICES AND MEDICINAL HERBS. An apparatus which allows the direct measurement of the antioxidant capacity of volatiles compounds emitted from some herbs and culinary spices is described. The device comprises: a sample chamber, a mixing chamber, a pump and, a detection system. Volatiles from Clove (Syzygium aromaticum (L.) Merr. \& L.M. Perry) were purged and captured into a DPPH-containing solution and changes in the absorbance were recorded on-line. Linear response was observed when temperature was set between $30-53^{\circ} \mathrm{C}$; nitrogen flow was $15 \mathrm{~mL} \mathrm{~min}^{-1}$ during $60 \mathrm{~min}$; DPPH concentration was $20 \mu \mathrm{mol} \mathrm{L}^{-1}$ and a sample size (powdered Clove) ranged between 200-1000 mg.
\end{abstract}

Keywords: antioxidants; DPPH; essential oils.

\section{INTRODUCCION}

Las plantas aromáticas y especias constituyen la principal fuente de aceites esenciales empleadas en la industria farmacéutica, cosmética y de alimentos. Tradicionalmente los aceites esenciales se han utilizado por sus propiedades como agentes aromatizantes. En los últimos años sin embargo, se ha puesto mucho interés en las propiedades anti-microbianas y antioxidantes que estos poseen ${ }^{1-3} \mathrm{El}$ efecto antioxidante de las plantas aromáticas resulta muy atractivo, debido a que en los alimentos ricos en lípidos insaturados, previenen o retardan el proceso de enranciamiento. Este efecto es importante, no sólo en términos de preservación, sino que además por sus potenciales beneficios para la salud. ${ }^{4}$ Los antioxidantes actúan como agentes estabilizadores de radicales libres inhibiendo la peroxidación lipídica. Este proceso esta involucrado en el desarrollo de varias enfermedades comunes, que incluyen a la aterosclerosis y desordenes neurodegenerativos como la enfermedad de Alzheimer., ${ }^{5,6}$

Durante mucho tiempo se ha recurrido a empleo de antioxidantes sintéticos para prevenir el deterioro oxidativo de los alimentos. No obstante, esta práctica se ha vuelto controversial dados los efectos tóxicos que estos pueden presentar. ${ }^{7}$ Esto último ha motivado la constante búsqueda de antioxidantes lipofílicos más seguros. De ahí, que sea de particular importancia el desarrollo de métodos para la evaluación de las potenciales propiedades antioxidantes de las plantas aromáticas y especias. Todos los métodos empleados consideran la obtención de un aceite esencial por extracción mediante hidrodestilación, solvente orgánico, microondas o fluidos supercríticos (SCF). Por lo tanto, hasta ahora, el proceso de extracción ha sido un paso previo necesario para estudio de la capacidad antioxidante, la que posteriormente puede ser estudiada en diferentes modelos. ${ }^{8-10}$ Uno de los radicales más empleados para ello es la especie coloreada 2,2-diphenil-1-picrilhidrazilo (DPPH). ${ }^{11-14}$ Generalmente, el método consiste en la mezcla de alícuotas del aceite esencial con el radical

\footnotetext{
*e-mail: edgar.pastene@gmail.com
}

DPPH con posterior registro de la variación de absorbancia en el tiempo. Si bien lo anterior se puede realizar en tubos de ensayo o micro-placas de 96 pocillos, algunas adaptaciones incluyen el uso del radical como agente de detección en placas de silicagel (TLC dot-blot), en análisis automatizado por inyección secuencial (SIA), como reactivo post-columna o parte de la fase móvil en HPLC..$^{15,16}$ Estas modificaciones permiten pesquisar en forma directa, cuáles serían las sustancias responsables del efecto antioxidante dentro de un extracto vegetal. En forma complementaria al uso del DPPH es habitual encontrar trabajos en los que se emplea el sistema $\beta$-caroteno/ ácido linoleico. Este método resulta un tanto más laborioso debido a la preparación de liposomas y los largos tiempos de oxidación a 50 ${ }^{\circ} \mathrm{C}$ requeridos para blanquear el $\beta$-caroteno. ${ }^{17-19}$

Uno de los principales problemas de los aceites esenciales es su limitada solubilidad en agua, lo que impide el uso de modelos en los cuales el radical se genera en un medio acuoso. Sin embargo, algunos investigadores han empleado con éxito el radical ácido 2,2'-azinobis3-etilbenzotiazolino-6-sulfónico (ABTS), diluido en etanol para la evaluación de la capacidad antioxidante de moléculas poco solubles. ${ }^{20}$ De igual manera, el empleo del ensayo Ferric Reducing Antioxidant Power (FRAP), puede ser una alternativa cuando las cantidades de aceite esencial empeladas son bajas. ${ }^{21} \mathrm{Si}$ bien la actividad antioxidante de algunas sustancias como el eugenol, isoeugenol, carvacrol y timol es muy marcada en los ensayos ABTS y FRAP, resulta interesante saber si estos compuestos volátiles pueden ejercer una acción antioxidante a las concentraciones que resultan de su emisión desde sus matrices naturales (por ejemplo, al encontrarse como parte de un polvo seco), y que eventualmente podrían alcanzarse en mucosas luego se su inhalación o ingesta. La emisión de los compuestos aromáticos desde una planta o alimento sigue en cada caso una cinética diferente, por lo que el efecto que estos tengan cuando alcanzan la mucosa nasal para ser inhaladas puede ser impredecible y no estar correlacionado con los resultados para el aceite puro. De la misma forma, los estudios in vitro realizados con diferentes aceites no permiten concluir que el efecto antioxidante será reproducido in vivo. ${ }^{22,23}$ 
Hasta ahora no existe un dispositivo que permita la evaluación directa de la capacidad antioxidante de volátiles emitidos de su fuente a temperaturas moderadas $\left(25-50{ }^{\circ} \mathrm{C}\right)$. En este trabajo se explora por primera vez la posibilidad de detectar esta propiedad para algunas sustancias antioxidantes en fase gaseosa provenientes de plantas aromáticas empleadas como agentes medicinales o especias de uso culinario. En particular, se analiza la emisión de antioxidantes volátiles desde clavo de olor (Syzygium aromaticum (L.) Merr. \& L.M. Perry). Esta especia contiene eugenol como principal sustancia de reconocida actividad antioxidante. El sistema presentado permite la detección directa de antioxidantes sin previa extracción del aceite esencial. Los volátiles emitidos, son capturados y sometidos a reacción con el radical libre estable DPPH en una sola operación. Nuestros resultados sugieren que para el caso del clavo de olor, se pueden alcanzar concentraciones antioxidantes efectivas dependiendo de la temperatura, la calidad y cantidad de materia prima que los emite.

\section{MATERIALES Y MÉTODOS}

\section{Materiales vegetales y reactivos}

Syzygium aromaticum (L.) Merr. \& L.M. Perry fue adquirido en un supermercado local. Carvacrol, timol, eugenol e isoeugenol, fueron obtenidos de Roth (Karlsruhe, Alemania); 2,2-difenil-1picrilhidrazilo (DPPH) fue obtenido de Sigma-Aldrich (Santiago, Chile). Otros solventes y reactivos fueron adquiridos de Merck (Darmstadt, Alemania).

\section{Determinación de polifenoles totales}

Se pesaron exactamente $100 \mathrm{mg}$ de la muestra pulverizada, la cual fue extraída tres veces con $25 \mathrm{~mL}$ de metanol $80 \%$ a temperatura ambiente y con agitación constante durante $30 \mathrm{~min}$. Los extractos filtrados fueron llevados a un volumen de $100 \mathrm{~mL}$. Para la determinación del contenido de fenoles totales, se tomó una alícuota de $50 \mu \mathrm{L}$ siguiendo un protocolo previamente publicado. ${ }^{24}$ La curva estándar se preparó a partir de soluciones de ácido gálico: 20-40-80-160-240-280 $\mathrm{mg} \mathrm{L}^{-1}\left(\mathrm{y}=0,0872 \mathrm{x}+0,0376 ; \mathrm{R}^{2}=0,9942\right)$.

\section{Determinación de eugenol en clavo por HPTLC}

El contenido de aceite esencial se determinó por hidrodestilación según recomendaciones de la Farmacopea Europea en aparato de clavenger con modificación para aceites esenciales de mayor densidad que el agua. El contenido de eugenol en el aceite esencial obtenido fue determinado por HPTLC de acuerdo a Pathak et al., con algunas modificaciones. ${ }^{25}$ Brevemente, se preparó una curva de calibración para eugenol aplicando 200-1000 ng $\mathrm{mL}^{-1}$. En placas HPTLC de silicagel F-254 (Merck). El desarrollo se realizó en cámara horizontal (Camag), con el solvente de arrastre tolueno-acetato de etilo-ácido fórmico $(3+2+0.4 \mathrm{v} / \mathrm{v})$ por una distancia de $5 \mathrm{~cm}$. Del aceite apropiadamente diluido se aplicó $2 \mu \mathrm{L}$ en triplicado. Las placas se secaron a temperatura ambiente y posteriormente fueron escaneadas a $280 \mathrm{~nm}$ utilizando el scanner Camag TLC 3 equipado con software CATS 4.0. De la curva de calibración (áreas), se calculó el contenido de eugenol en el aceite y estimó la cantidad libre en la muestra pulverizada.

\section{Determinación de la actividad antioxidante con $2,2^{\prime}$-difenil-1- picrilhidrazilo (DPPH)}

Como método de referencia, la actividad antioxidante el aceite esencial extraído del clavo de olor fue medida en términos de su capacidad de donar hidrogeno o atrapar radicales libres, usando el radical libre estable DPPH según la metodología de Politeo et al., ${ }^{26}$ con algunas modificaciones. En breve, se preparó una serie de diluciones en del aceite esencial en metanol desde 8 hasta $0,008 \mathrm{~g} \mathrm{~L}^{-1}$. Utilizando placas de 96 pocillos, una alícuota de las muestras $(10 \mu \mathrm{L})$ fue mezclada con una solución $100 \mu \mathrm{mol} \mathrm{L}^{-1}$ de DPPH en metanol. La decoloración del radical DPPH fue registrada inmediatamente a 517 nm durante 45 min, utilizando un multi-lector Synergy HT (Biotek, USA). Todas las determinaciones fueron realizadas en triplicado. El porcentaje de decoloración del radical DPPH por el aceite de clavo de olor se calculó a partir de las gráficas cinéticas de decoloración, para las que se calculó el área bajo la curva $(\mathrm{ABC})$ según las ecuaciones publicadas previamente por Cheng et al.. ${ }^{27}$

\section{Determinación del rango de trabajo}

Se preparó soluciones de las sustancias patrón en cloroformo en una concentración de $60 \mathrm{mmol} \mathrm{L}^{-1}$. A partir de esta solución se tomó alícuotas entre $0,2-40 \mu \mathrm{L}$ y diluyeron con radical $\mathrm{DPPH}$, hasta un volumen de $8 \mathrm{~mL}$ (volumen total que será utilizado en el sistema de detección) para lograr concentraciones finales entre 1,5-300 $\mu \mathrm{mol} \mathrm{L} \mathrm{L}^{-1}$. El análisis fue realizado en triplicado y la absorbancia de las muestras comenzó a registrarse inmediatamente por un periodo de $30 \mathrm{~min}$. Las curvas de porcentaje de decoloración fueron transformadas utilizando el logaritmo de la concentración final de antioxidante. De esta forma se obtuvo la $\mathrm{EC}_{50}$ para cada sustancia patrón. $\mathrm{La} \mathrm{EC}_{50}$ se define como la concentración necesaria que debe alcanzarse en la solución del radical para producir una decoloración equivalente a un $50 \%$ de la absorbancia inicial. En un experimento adicional se registró el espectro UV-VIS de soluciones correspondientes a las mismas concentraciones, en ausencia de radical. Para cada sustancia se determinó la longitud de máxima absorción y el coeficiente de extinción molar, con el que se construyó curvas de calibración para la estimación directa del contenido de antioxidante capturado efectivamente por el solvente.

\section{Preparación del radical DPPH}

El radical DPPH fue preparado en cloroformo a una concentración de $100 \mu \mathrm{mol} \mathrm{L}^{-1}$ y diluido con el mismo solvente hasta una absorbancia de 0,200 (concentración final de $20 \mu \mathrm{mol} \mathrm{L}^{-1}$ ). Esta solución se preparó diariamente.

\section{Descripción del sistema}

El aparato para la captura y reacción de volátiles con el radical DPPH se esquematiza el material suplementario Figura 1S. Este consta de las siguientes partes: 1) bombona de nitrógeno con regulador de flujo $(15 \mathrm{~mL}$ $\mathrm{min}^{-1}$ ), en conexión con la cámara o contenedor de la muestra fabricado de acero inoxidable y conectado por una línea del mismo material (20 $\mathrm{cm} x$ 1,6 mm o.d. x 0,8 mm d.i.). La cámara de muestra fue construida de acero inoxidable con dimensiones ajustables según tamaño de la muestra (50-5000 mg). La cámara posee una línea de salida (20 cm x 1,6 mm o.d. x $0,8 \mathrm{~mm}$ d.i.), que lleva los volátiles hacia el reactor. 2) Reactor de PTFE para reacción de volátiles con DPPH, posee temperatura controlada mediante una banda calefactora con regulador Normschliff Geratebau (Wertheim, NGW-TRII, Germany) y un volumen total de reacción de $8 \mathrm{~mL}$. El reactor esta constituido de tres partes: a) cámara de difusión donde ocurre la primera etapa de mezclado entre los volátiles y el radical $(0,5 \mathrm{~mL}) ; \mathrm{b})$ cámara de mezclado donde ocurre una segunda etapa de mezclado con mayor eficiencia $(2,5 \mathrm{~mL})$; c) cámara de muestreo y ventilación permite llevar un volumen de la mezcla hacia el sistema de detección y a la vez deja salir gases que no reaccionan (1,5 mL). 3) Bomba de doble pistón Lachrom (Merck-Hitachi), succiona en forma 
continua un volumen de la mezcla desde el reactor llevándolo hacia un espectrofotómetro (Unicam He $\lambda$ ios $\alpha$ ) con celda de flujo continuo Hellma (volumen de $100 \mu \mathrm{L}$, paso óptico de $100 \mathrm{~mm}$ ). La conexión de salida y entrada de la bomba están hechas mediante líneas de teflón (volumen total 3,5 mL). Esta conexión permite retornar una vez más la solución hacia la cámara de mezclado con un flujo fijo de 7,0 mL $\min ^{-1}$ (presión total 85 psi). 4) PC con software Vision 32, para registro de los datos en línea.

\section{Procedimiento para el análisis de la capacidad antioxidante de volátiles emitidos directamente de una fuente vegetal}

\section{Incubación}

Las muestras introducidas en la cámara herméticamente cerrada se sumergen en un baño termoregulado por $1 \mathrm{~min}$, sin flujo de nitrógeno y con el flujo de radical detenido.

\section{Radical DPPH}

Previo a la introducción de la muestra se debe circular un volumen de $8 \mathrm{~mL}$ de radical el cual se carga por en el tubo de ventilación. El sistema se llena con un flujo de $9.90 \mathrm{~mL} \mathrm{~min}^{-1}$. Para el análisis, el

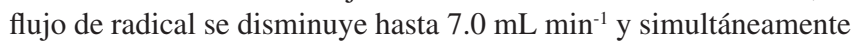
se abre el paso de nitrógeno a través de la muestra.

\section{Medición}

Para cada medición se monitorea la estabilidad del radical. La absorbancia se registra a $517 \mathrm{~nm}$ durante $30 \mathrm{~min}$ y se expresa como porcentaje de DPPH remanente en la solución.

\section{Drenaje del reactor}

Se drena cuidadosamente el radical usado por la compuerta de entrada de radical. El flujo de radical se detiene y deja solamente burbujear nitrógeno durante unos $30 \mathrm{~s}$. Se cierra el paso de nitrógeno y retira la cámara de muestra del baño termoregulado. Una vez enfriada, la cámara es abierta y se procede a retirar la muestra.

\section{Limpieza del sistema}

La cámara es lavada secuencialmente con detergente (común de laboratorio), etanol y cloroformo. Si se analizan muestras con un alto contenido de volátiles, es necesario someter la cámara a un calentamiento de $150{ }^{\circ} \mathrm{C}$ durante $30 \mathrm{~min}$. La cámara se deja enfriar a temperatura ambiente y se seca muy bien antes de ser usada nuevamente. Para la limpieza del sistema de captura, se agrega $10 \mathrm{~mL}$ de metanol por el tubo de ventilación. Se conecta rápidamente la línea de entrada y deja operar el equipo por $10 \mathrm{~min}$ a flujo máximo $\left(9,9 \mathrm{~mL} \mathrm{~min}^{-1}\right)$. Se drena de la misma forma y repite la operación con $10 \mathrm{~mL}$ de cloroformo. Durante estas operaciones la cámara de muestra es removida para su limpieza y el suministro de nitrógeno permanece cerrado y desconectado. Esta operación genera un eficiente retro-lavado de la línea de acero inoxidable que estuvo en contacto con los volátiles, permitiendo la remoción de sustancias remanentes. Una vez finalizada esta etapa, y antes de volver a colocar otra muestra, se corrió un blanco de radical para asegurar la correcta limpieza del equipo. Por convención se consideró aceptable una disminución del blanco no mayor que un $2 \%$. Cuando este valor es mayor, se inicia un nuevo ciclo de lavado.

\section{Efecto de la temperatura sobre la emisión de volátiles antioxidantes}

Para este ensayo se introduce $500 \mathrm{mg}$ de clavo de olor. Posterior a la introducción en la cámara de muestra, se registra los cambios de absorbancia con diferentes temperaturas de incubación $\left(25-80{ }^{\circ} \mathrm{C}\right)$. Para cada temperatura se corre un blanco y analiza en triplicado.

\section{RESULTADOS Y DISCUSION}

\section{Caracterización de la muestra de clavo de olor}

La muestra presentó un contenido de fenoles totales expresados como ácido gálico (GAE) de 23,6\% (236 $\left.\mathrm{mg} \mathrm{g}^{-1}\right)$. El contenido de aceite esencial, obtenido por hidrodestilación de la planta, fue de un $11,0 \%(\mathrm{v} / \mathrm{m})$. El análisis por HPTLC de éste aceite esencial arrojó como resultado un $82 \%$ de eugenol. Este valor esta dentro de las especificaciones de la Farmacopea Europea para esta planta y con él se estimó un contenido de eugenol de 9,02\% (en base seca). Del resultado anterior se puede deducir que un $62 \%$ de los compuestos capaces de reducir al reactivo de Folin-Ciocalteu corresponden a sustancias no volátiles. El 38\% de los compuestos volátiles restante incluye principalmente al eugenol. Debido a esto se decidió utilizar la equivalencia en eugenol ( $\mu$ moles $\mathrm{g}^{-1}$ ), en lugar de los miligramos de polvo de la especia. La dependencia de la capacidad antioxidante en función de la masa de muestra se comprobó utilizando como método de comparación la medición de la decoloración del radical DPPH inducida por distintas diluciones del aceite esencial de clavo de olor. Con los gráficos obtenidos (Figura 2S) se calculó una $\mathrm{EC}_{50}$ de $226 \mathrm{mg} \mathrm{L}^{-1}\left(\mathrm{R}^{2}=0,9971\right)$. En el trabajo de Politeo el al., ${ }^{26} \mathrm{la} \mathrm{EC}_{50}$ obtenida para el aceite esencial de Ocimum basilicum L., fue de 1378 $\mathrm{g} \mathrm{L}^{-1}$. Este valor refleja la concentración de volátiles con propiedades antioxidantes en esta especie es inferior a la encontrada en el calvo de olor. En efecto, los mismos autores reportan un porcentaje de eugenol de 5,9\%, el que en el aceite de $O$. basilicum equivale a unos $0,09 \mathrm{~g} \mathrm{~L}^{-1}$ de eugenol. En las condiciones utilizadas en el estudio indicado, la equivalencia de eugenol para $O$. basilicum, corresponde casi exactamente a la $\mathrm{EC}_{50}\left(0,096 \mathrm{~g} \mathrm{~L}^{-1}\right)$ del eugenol puro.

\section{Determinación del rango de trabajo}

Para establecer el peso de muestra a emplear en el estudio, se construyó curvas de decoloración de DPPH con diferentes concentraciones de eugenol. En la Figura 1a se muestra las curvas registradas durante 30 min para las concentraciones $\mathrm{EC}_{50}$ de timol, isoeugenol y carvacrol, que fueron comparadas con la de eugenol. La $\mathrm{EC}_{50}$ de eugenol calculada en nuestras condiciones de trabajo $\left(20 \mu \mathrm{mol} \mathrm{L}{ }^{-1}\right.$ de DPPH, $8 \mathrm{~mL}$ volumen final), a partir de las gráficas mostradas en la Figura $1 \mathrm{~b}$, fue de $135 \mu \mathrm{mol} \mathrm{L} \mathrm{L}^{-1}$. Comparativamente, dicha concentración es menor a la obtenida para timol y carvacrol (775 y 440 $\mu \mathrm{mol} \mathrm{L}{ }^{-1}$, respectivamente). La concentración mínima de eugenol que generó un cambio detectable de la absorbancia del DPPH (mayor que un 5\%), fue de 1,5 $\mu \mathrm{mol} \mathrm{L} \mathrm{L}^{-1}$. Para isoeugenol se obtuvo una $\mathrm{EC}_{50} \mathrm{de}$ $22 \mu \mathrm{mol} \mathrm{L}{ }^{-1}$, seis veces menor que para eugenol y la concentración mínima que generó un cambio en la absorbancia del radical DPPH fue de $0,6 \mu \mathrm{mol} \mathrm{L}{ }^{-1}$. Los resultados son coherentes con reportes previos para estos compuestos al ser medidos en solventes alcohólicos como metanol y etanol. ${ }^{28}$ Sin embargo, no siempre la magnitud de $\mathrm{EC}_{50}$ coincide con los publicados por otros autores. ${ }^{14}$ La razón de esta discrepancia radica en que nuestras mediciones fueron realizadas en un medio más apolar $\left(\mathrm{CHCl}_{3}\right)$ lo que reduce el efecto de los grupos $\mathrm{R}-\mathrm{OH}$ sobre la velocidad de la reacción del DPPH con los fenoles de la muestra. ${ }^{29}$ Isoeugenol es un antioxidante muy similar al eugenol pero, su reactividad es mucho mayor debido a que la disposición del grupo hidroxilo en posición orto con un metoxilo permite la formación de un puente hidrógeno intramolecular, el cual es energéticamente favorable con una magnitud de $3,74 \mathrm{kcal} / \mathrm{mol} .{ }^{30}$ Comparativamente, este valor es menor al de otros antioxidantes como el ácido ferúlico $(3,91 \mathrm{kcal} / \mathrm{mol})$ y el alcohol coniferílico $(3,93 \mathrm{kcal} / \mathrm{mol})$, lo que facilita la donación hacia radicales libres. ${ }^{30}$

En nuestro estudio se estableció que para obtener una respuesta 
detectable en el estudio con la planta se requiere capturar sobre 2 $\mu \mathrm{mol} \mathrm{L} \mathrm{L}^{-1}$ de eugenol en los $8 \mathrm{~mL}$ de la solución de DPPH presente en el reactor. De acuerdo a los resultados anteriores la planta contiene un $9,02 \%$ de eugenol, lo que equivale a $549 \mu \mathrm{mol} \mathrm{g} \mathrm{g}^{-1}$. Por lo tanto, introduciendo $500 \mathrm{mg}$ de planta en el sistema, en teoría se esperaría llegar a porcentajes de decoloración superiores al 50\%. Sin embargo, dado el alto punto de ebullición de los compuestos fenólicos en un aceite esencial $\left(256{ }^{\circ} \mathrm{C}\right.$ para eugenol) en comparación con los monoterpenos y los hidrocarburos, es muy probable que a bajas temperaturas la remoción de eugenol libre diste de ser completa. Teniendo presente lo anterior, se decidió hacer un primer estudio utilizando el sistema diseñado para la captura de volátiles, con el fin de conocer el efecto de la temperatura sobre la emisión de eugenol desde clavo de olor.

\section{Efecto de la temperatura sobre la emisión de volátiles antioxidantes}

La emisión de volátiles desde una fuente vegetal depende de la temperatura. Así, se observa en la Figura 2 que la emisión de antioxidantes volátiles es dependiente de la temperatura hasta los $50{ }^{\circ} \mathrm{C}$.
A
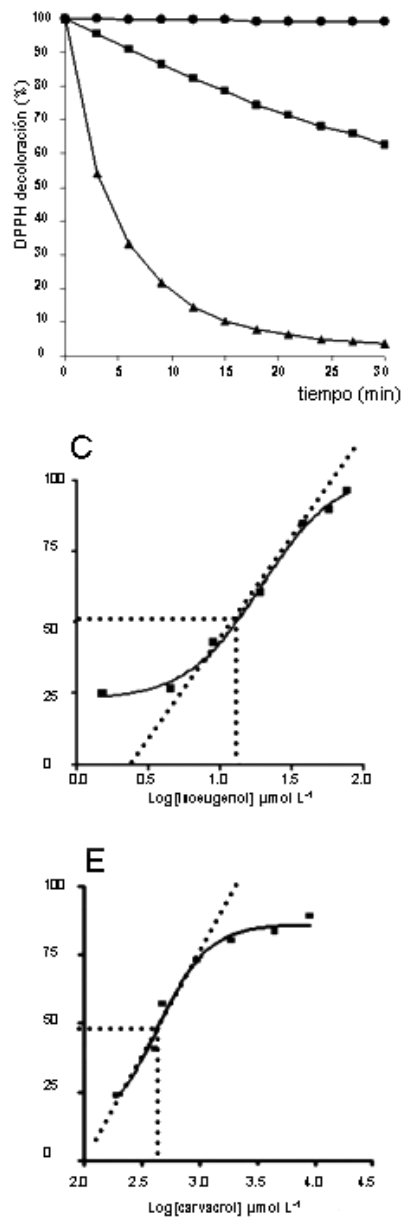

B

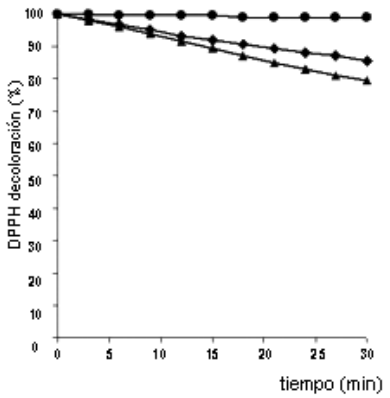

D
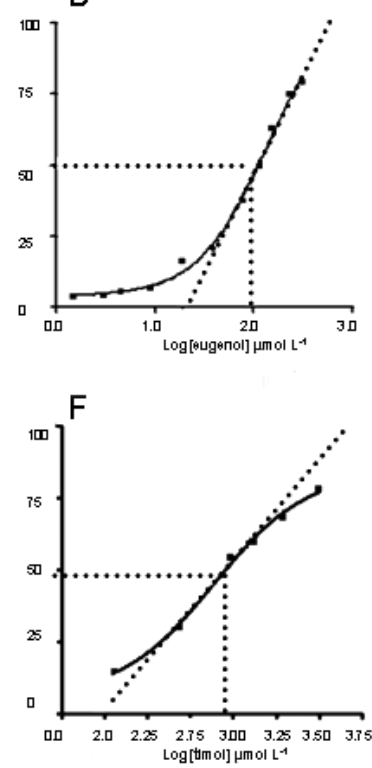

Figura 1. Efecto comparativo de diferentes antioxidantes volátiles sobre la cinética de decoloración del radical DPPH. $2 A$ : control (•); isoeugenol $40 \mu \mathrm{mol} \mathrm{L} L^{-1}(\boldsymbol{\Delta})$; eugenol $40 \mu \mathrm{mol} \mathrm{L} L^{-1}(\bullet)$. 2B: control (•); carvacrol 200 umol $L^{-1}(\mathbf{\Delta})$; y timol $200 \mu \mathrm{mol} L^{-1}(\bullet)$. $2 \boldsymbol{C}$ - $\boldsymbol{F}$ : gráficas de porcentaje de decoloración versus log[antioxidante] para la determinación de la $E C_{50}$. Todas las mediciones se realizaron con una solución de DPPH $20 \mu \mathrm{mol} L^{-1}$ y durante $60 \mathrm{~min}$

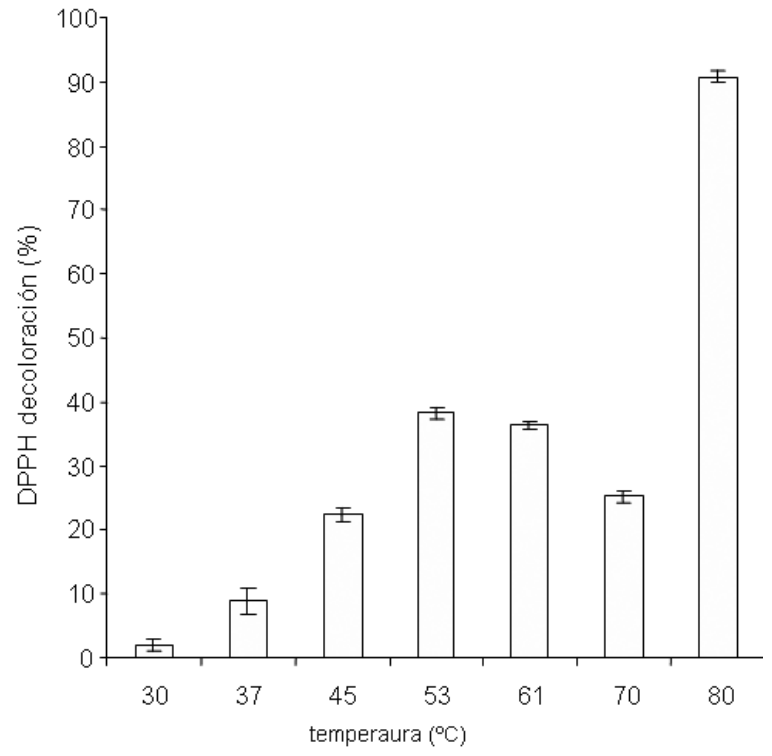

Figura 2. Decoloración del radical libre estable DPPH por volátiles emitidos desde clavo de olor a diferentes temperaturas (media $\pm S D, n=3)(p<0.05)$. La cantidad de muestra fue de $500 \mathrm{mg}$. Todas las mediciones se realizaron con una solución de DPPH 20 mol $L^{-1}$ y durante $60 \mathrm{~min}$

En nuestro estudio, ya a temperaturas mayores de $37{ }^{\circ} \mathrm{C}$ es posible detectar cambios en la absorbancia del DPPH que no son evidentes a temperatura ambiente $\left(25^{\circ} \mathrm{C}\right)$. Analíticamente, la presencia de eugenol se comprobó midiendo las bandas de absorbancia UV de soluciones de captura sin radical (cloroformo) y calculando la concentración de antioxidante efectivamente capturado desde la fuente de emisión. El espectro ultravioleta de esta solución posee las bandas de absorción características del eugenol (210 y $270 \mathrm{~nm})$. Se observa que a $37^{\circ} \mathrm{C}$ la extracción de eugenol corresponde a un $2 \%$ de la cantidad presente en la muestra pulverizada $(500 \mathrm{mg})$, lo que basta para obtener una decoloración superior al 5\% a los $60 \mathrm{~min}$. Usando la misma cantidad de muestra y $45^{\circ} \mathrm{C}$ el porcentaje de extracción llegó al 9\% a los $30 \mathrm{~min}$ y a un $22 \%$ a los $60 \mathrm{~min}$. Con el uso de temperaturas superiores (hasta $80^{\circ} \mathrm{C}$ en este estudio), la extracción de antioxidantes se vio modificada dramáticamente, puesto que se generó un cambio de pendiente en la curva de decoloración del DPPH. Esto altera la tendencia observada para el caso del patrón de eugenol puesto que, a concentraciones mayores en el medio, no se produce el mismo cambio brusco pendiente. En el caso de la muestra, la pendiente y la absorbancia final conservan una relación casi lineal en función de la temperatura sólo en el rango de $\operatorname{los} 30-53{ }^{\circ} \mathrm{C}(\mathrm{y}=1,5957 \mathrm{x}-47,919$; $\left.\mathrm{R}^{2}=0,984\right)$. Lo anterior, no puede atribuirse a una inestabilidad del radical, puesto que los blancos respectivos se muestran estables durante todo periodo de análisis, sin producirse una decoloración significativa ( $>5 \%$ ), por efecto del contacto con vapores a mayor temperatura. El cambio observado puede tener varias causas, dentro de las cuales se puede mencionar el mayor punto de ebullición que poseen otros antioxidantes derivados del fenilpropano como el isoeugenol $\left(268^{\circ} \mathrm{C}\right)$, timol $\left(232^{\circ} \mathrm{C}\right)$ y carvacrol $\left(237^{\circ} \mathrm{C}\right)$. Esta propiedad hace que su recuperación desde una muestra seca sea poco eficiente en comparación con la extracción forzada por agua hirviente, solventes orgánicos o fluidos supercríticos. En el caso particular del eugenol, este posee un punto de ebullición inferior al de isoeugenol. Según lo mostrado en la Figura 1, el isoeugenol posee una $\mathrm{EC}_{50}$ muy baja y alta reactividad. Por lo tanto, bastarían concentraciones del orden de $1 \mu \mathrm{mol} \mathrm{L} \mathrm{L}^{-1}$ en el reactor para producir una decoloración del radical DPPH mayor al 5\%. En el aceite de clavo de olor, la concentración 
de isoeugenol está entre un 5-10\%, lo que corresponde a 33-66 $\mu$ mol $\mathrm{g}^{-1}$ de de planta. Por lo tanto, bastaría con poder arrastrar un $9 \%$ de esta concentración, para lograr una decoloración del radical DPPH mayor al $90 \%$. En efecto, a $80{ }^{\circ} \mathrm{C}$ se puede apreciar que el porcentaje de decoloración llega al 91\%, lo que es coherente con su mayor punto de ebullición y eficiencia como antioxidante.

La incorporación de agua al estado de vapor puede resultar con seguridad en un mayor tenor de extracción debido a que esta aumenta la humectación de los tejidos vegetales permitiendo la salida del aceite esencial o las reacciones de hidrólisis que liberan volátiles a partir de precursores como glicósidos y ésteres. Sin embargo, nosotros omitimos este procedimiento por no ser relevante para los objetivos del presente estudio. Interesantemente, el hallazgo de antioxidantes de mayor volatilidad y de menor polaridad como el terpinoleno (PM $=136,23$; punto de ebullición $\left.185^{\circ} \mathrm{C}\right)$ y el $\gamma$-terpineno $(\mathrm{PM}=136,24$; punto de ebullición: $183^{\circ} \mathrm{C}$ ) hace pensar en otras futuras aplicaciones del sistema diseñado, para la evaluación de esencias con efecto antioxidante en sustratos menos polares. Sin embargo, se debe consignar que las propiedades antioxidantes de estos volátiles no-fenólicos en sistemas biológicos (LDL) tienen que ver con la protección de la oxidación $\beta$-caroteno y al desplazamiento del $\alpha$-tocoferol hacia la superficie de las lipo-partículas. ${ }^{31}$ De esta forma, dichos compuestos actuarían como coadyuvantes (sinergia) en la protección frente al daño por ROS. No obstante, algunos autores señalan que estas sustancias son capaces, además de proteger al -caroteno en el ensayo de blanqueamiento, de decolorar al radical DPPH al igual que los derivados de fenilpropano. ${ }^{32}$

\section{Efecto de la masa en la capacidad antioxidante}

En la Figura 3 se muestra que la decoloración del radical DPPH depende de la masa de planta presente en el sistema (equivalentes de eugenol). La dependencia se observa entre 200 y $1000 \mathrm{mg}$ de planta (110 y $550 \mu \mathrm{mol} \mathrm{g}{ }^{-1}$ equivalentes de eugenol). Tanto a menores (< de $100 \mathrm{mg}$ ) como mayores (> $1200 \mathrm{mg}$ ) cantidades de planta, hubo pérdida del comportamiento lineal de la emisión de antioxidantes. Con

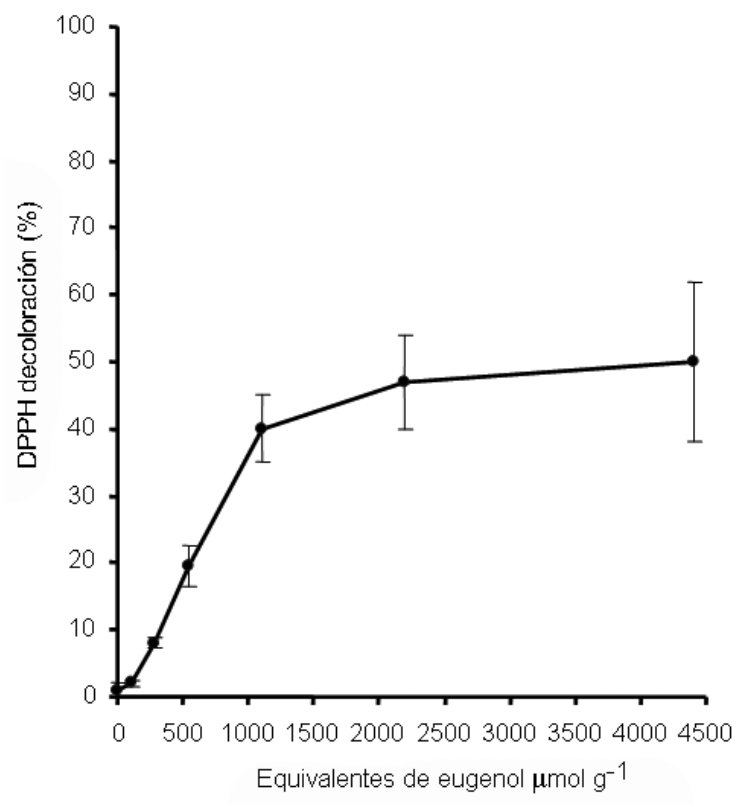

Figura 3. Efecto de diferentes cantidades de clavo de olor sobre la decoloración del radical radicales DPPH (media $\pm S D, n=3$ ). Todas las mediciones

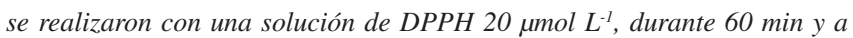
$37^{\circ} \mathrm{C}$ mayores cantidades, esta pérdida de dependencia puede ser atribuida a alteraciones en el flujo de gas nitrógeno a través de la muestra, o bien a un fenómeno de condensación de humedad. Es esperable que frente a variaciones en la temperatura y cantidad de muestra, cada planta presente un comportamiento diferente. De la misma manera, así como la temperatura puede influir en la composición de la mezcla gaseosa que reaccionará con el radical DPPH, no se puede descartar la existencia de un efecto antioxidante sinérgico como el mencionado anteriormente..$^{30}$ Como ejemplo, al estudiar una serie de aceites esenciales obtenidos de representantes del género Lamiaceae (Ocimun basilicum; Origanum vulgare y Thymus vulgaris), se logró identificar varios compuestos con efecto antioxidante. Para ello se empleó DPPH, degradación de la 2-desoxyribosa por radical hidroxilo y el ensayo de TBA para estimar la lipoperoxidación de una mezcla comercial ("PRO-LIPO S") inducida por $\mathrm{Fe}^{++}$ascorbato y $\mathrm{Fe}^{++} / \mathrm{H}_{2} \mathrm{O}_{2}$. En $O$. basilicum, carvacrol, metil chavicol y una mezcla de hidrocarburos mono y sesquiterpénicos mostraron un claro efecto antioxidante. En $O$. vulgare y $T$. vulgaris se observó adicionalmente la presencia de timol como compuesto antioxidante de relevancia. ${ }^{33}$

La composición de volátiles variará significativamente si es que una parte importante de estos se encuentra como glicósido. Así, por ejemplo el efecto antioxidante del aceite esencial de nuez moscada (Myristica fragans Houtt.), es muy diferente al de sus aglicones volátiles liberados por hidrólisis. Se estimó que la diferencia en el efecto antioxidante estaba dada por la composición de ambos extractos. En el aceite esencial se identificó 10 compuestos mayoritarios (98\% del total), presentando sólo 2 en común con los volátiles liberados desde los aglicones, el eugenol y terpinen-4-ol. En los volátiles liberados de la fracción glicosídica se observó cerca de un $46 \%$ de isoeugenol. Este compuesto, que no se encontró en el aceite esencial, daría cuenta de la mayor capacidad antioxidante de la fracción glicosídica en los ensayos FRAP y DPPH. ${ }^{34}$ Como la composición de volátiles obtenidos de una misma planta puede ser variable, se refuerza la necesidad de investigar si las propiedades antioxidantes de un aceite esencial difieren o se asemejan a las de los volátiles que se emiten directamente desde una matriz vegetal.

\section{CONCLUSIONES}

En el presente trabajo se describe la construcción de un sistema para detección de antioxidantes volátiles y su aplicación al clavo de olor, habitualmente citado como especia de uso culinario y medicinal. El empleo de una solución $20 \mu \mathrm{mol} \mathrm{L}^{-1}$ de radical coloreado DPPH fue suficiente para la detección de estos antioxidantes en un medio orgánico de baja polaridad (cloroformo) no afectado por la temperatura del gas de arrastre. Las condiciones óptimas de operación se observaron entre 30 a $53{ }^{\circ} \mathrm{C}$, un flujo de arrastre de nitrógeno de $15 \mathrm{~mL} \mathrm{~min}^{-1}$ para un registro durante $60 \mathrm{~min}$. El tamaño de muestra que puede ser manejado sin generar problemas de oclusión y pérdida de una respuesta reproducible fue de 200 a $1000 \mathrm{mg}$ de clavo de olor. En ese rango la decoloración del radical DPPH fue proporcional a la masa de muestra. El sistema puede ser empleado para similares estudios en otras especies vegetales no solo como uso en la preservación de alimentos sino también por sus propiedades potencialmente beneficiosas para la salud.

\section{MATERIAL SUPLEMENTARIO}

El material suplementario está disponible gratuitamente en http:// quimicanova.sbq.org.br, en archivo de formato tipo PDF: Figura 1S. Gráfico representativo del efecto de diferentes diluciones del aceite esencial de Clavo de olor sobre la cinética de decoloración del radical DPPH. Figura 2S. Esquema del sistema para detección de antioxidantes volátiles emitidos desde fuentes vegetales. 


\section{REFERENCIAS}

1. Hinneburg, H.J.; Dorman, D.; Hiltunen, R.; Food Chem. 2006, 97, 122.

2. Hirasa, K.; Takemasa, M.; Spice science and technology, Dekker Inc.: New York, 1998.

3. Madsen, H. L.; Bertelsen, G.; Trends Food Sci. Technol. 1995, 6, 271.

4. Takao, T.; Kiatani, F.; Watanabe, N.; Yagi A.; Sakata K.; Biosci., Biotechnol., Biochem. 1994, 58, 1780.

5. Glass, C. K.; Witztum, J. L.; Cell. 2001, 23, 503.

6. Butterfield, D. A.; Lauderback, C. M.; Free Radical Biol. Med. 2002 , 32,1050 .

7. Cornwell, D. G.; Jones, K. H.; Jiang, Z.; Lantry, L. E.; Southwell, K. P.; Kohar, I.; Lipids. 1998, 33, 295

8. Ferhat, M. A.; Meklati, B. Y.; Smadja, J.; Chemat, F.; J. Chromatogr., A. 2006, 1112, 121 .

9. Lucchesi, M. E.; Chemat, F.; Smadja, J.; J. Chromatogr., A. 2004, 1043, 323.

10. Yepez, B.; Espinosa, M.; López, S.; Bolaños, G.; Fluid Phase Equilib. 2002, 194, 879

11. Chandrasekar, D.; Madhusudhana, K.; Ramakrishna, S.; Diwan, P. V.; J. Pharm. Biomed. Anal. 2006, 40, 460.

12. Zaeoung, S.; Plubrukarn A.; Keawpradub, N.; Songklanakarin J. Sci. Technol. 2005, 27, 801.

13. Jukic, M.; Milos, M.; Croat. Chem. Acta 2005, 78, 105.

14. Molyneux, P.; Songklanakarin J. Sci. Technol. 2004, 26, 211.

15. Kosar, M. D.; Dorman, H. J.; Can Baser, K. H.; Hiltunen, R.; J. Agric. Food Chem. 2004, 52, 5004.

16. Polasec, M.; Skata, P.; Opletal, L.; Jahodar, L.; Anal. Bioanal. Chem. 2004, 379, 754 .
17. Tepe, B.; Akpulat, H. A.; Sokmen, M.; Daferera, D.; Yumrutas, O.; Aydin, E.; Polissiou, M.; Sokmen, A.; Food Chem. 2006, 97, 719.

18. Kaur, C.; Kapoor, H. C.; Int. J. Food Sci. Technol. 2002, 37, 153.

19. Emmons, C. L.; Peterson, D. M.; Cereal Chem. 1999, 76, 902.

20. Nilsson, J.; Pillai, D.; Önning, G.; Persson, C.; Nilsson, Ä.; Akesson, B.; Mol. Nutr. Food Res. 2005, 49, 239.

21. Thaipong, K.; Boonprakob, U.; Crosby, K.; Cisneros-Zeballos, L.; Byrne, D. H.; J. Food Composition and Analysis 2006, 19, 669.

22. Gra mann, J.; Hippeli, S.; Dornisch, K.; Rohnert, U.; Beuscher, N.; Elstner, E. F.; Drug Res. 2000, 50, 135.

23. Gra mann; J.; Hippeli, S.; Spitzenberger, R.; Elstner, E. F.; Phytomedicine 2005, 12, 416.

24. Singleton, V. L.; Rossi J. A.; Am. J. Enol. Vitic. 1965, 16, 144.

25. Pathak, S. B.; Niranjan, K.; Padh, H.; Rajan, M.; Chromatographia 2004, 60, 241

26. Politeo, O.; Jukic, M.; Milos, M.; Food Chem. 2007, 101, 379.

27. Cheng, Z.; Moore, J.; Yu, L. L.; J. Agric Food Chem. 2006, 54, 7429.

28. Tominaga, H.; Kobayashi, Y.; Goto, T.; Kasemura, K.; Nombra, M.; Yakugaku Zasshi 2005, 125, 371.

29. Litwinienko, G.; Ingold, K. U.; J. Org. Chem. 2003, 68, 3433.

30. Nenadis, N.; Zhang, H. Y.; Tsimidou, M. Z.; J. Agric. Food Chem. 2003, $51,1874$.

31. Milde, J.; Elstner, E. F.; Gra mann, J.; Phytomedicine 2004, 11, 105.

32. Choi, H. S.; Song, H. S.; Ukeda, H.; Sawamura, M.; J. Agric. Food Chem. 2000, 48, 4156.

33. Bozin, B.; Mimica-Dukic, N.; Simin, N.; Anackov, G.; J. Agric. Food Chem. 2006, 54, 1822 .

34. Jukic, M.; Politeo, O.; Milos, M.; Croat. Chem. Acta 2006, 79, 209. 
UN SISTEMA PARA LA DETECCION DE ANTIOXIDANTES VOLATILES COMUNMENTE EMITIDOS DESDE ESPECIAS Y HIERBAS MEDICINALES

Edgar Pastene*, Maritza Gómez y Hernán Speisky

Laboratorio de Antioxidantes, Instituto de Nutrición y Tecnología de los Alimentos, Universidad de Chile, Macul 5540, Macul Santiago, Chile

Luís Núñez-Vergara

Departamento de Química Farmacológica y Toxicológica, Facultad de Ciencias Químicas y Farmacéuticas, Universidad de Chile, Olivos 1007, Independencia, Santiago - Chile

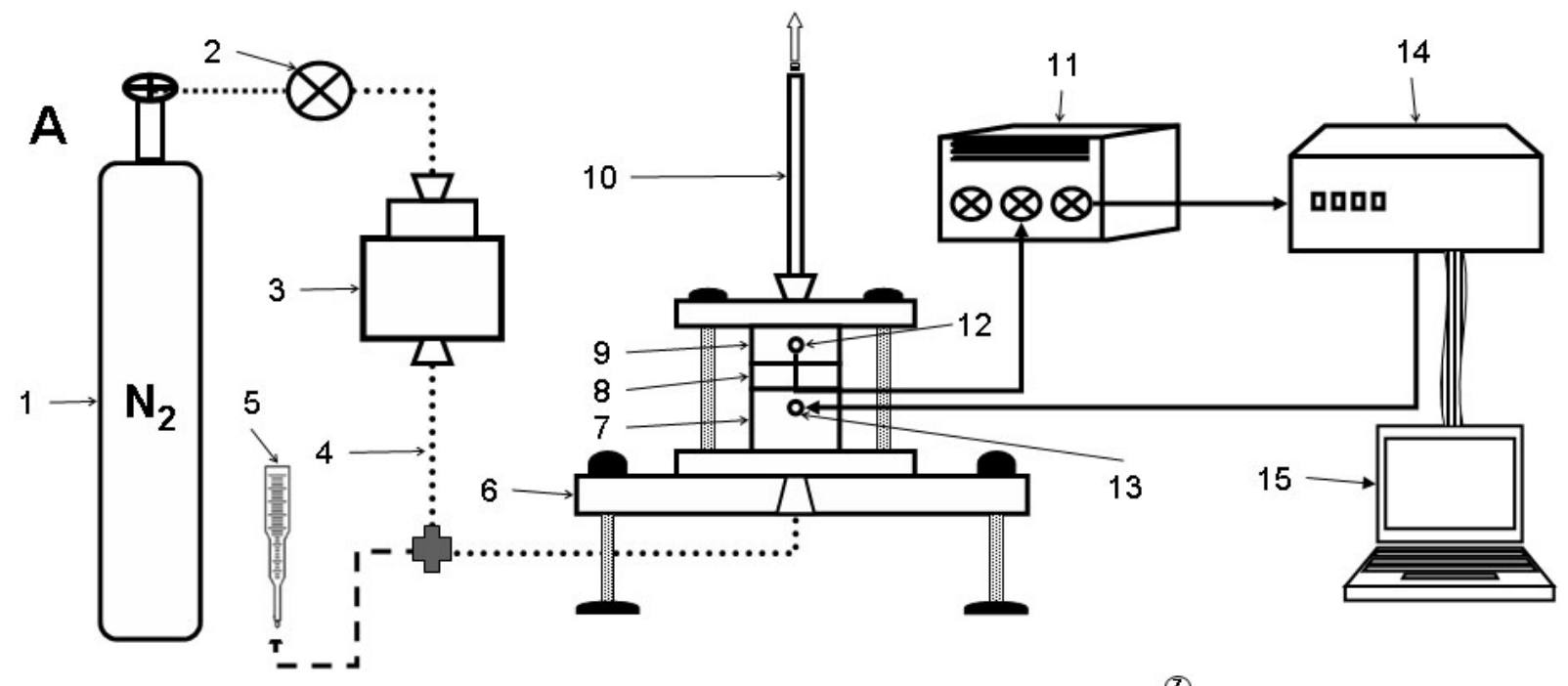

B
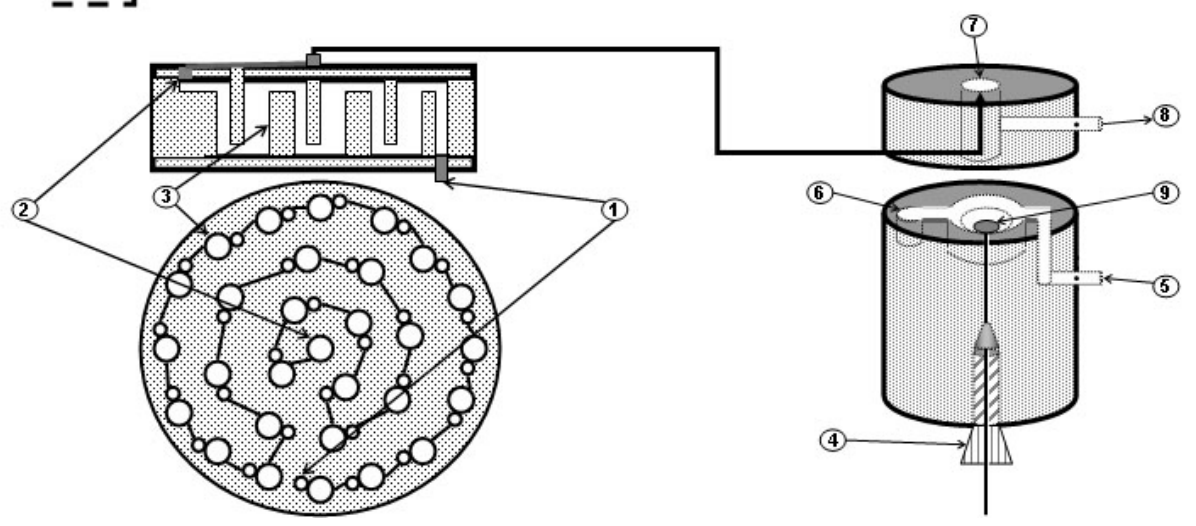

Figura 1S. A) Gráfico representativo del efecto de diferentes diluciones del aceite esencial de clavo de olor sobre la cinética de decoloración del radical DPPH. B) Gráfica semi-log de la concentración de aceite de clavo versus el porcentaje de decoloración del radical DPPH 

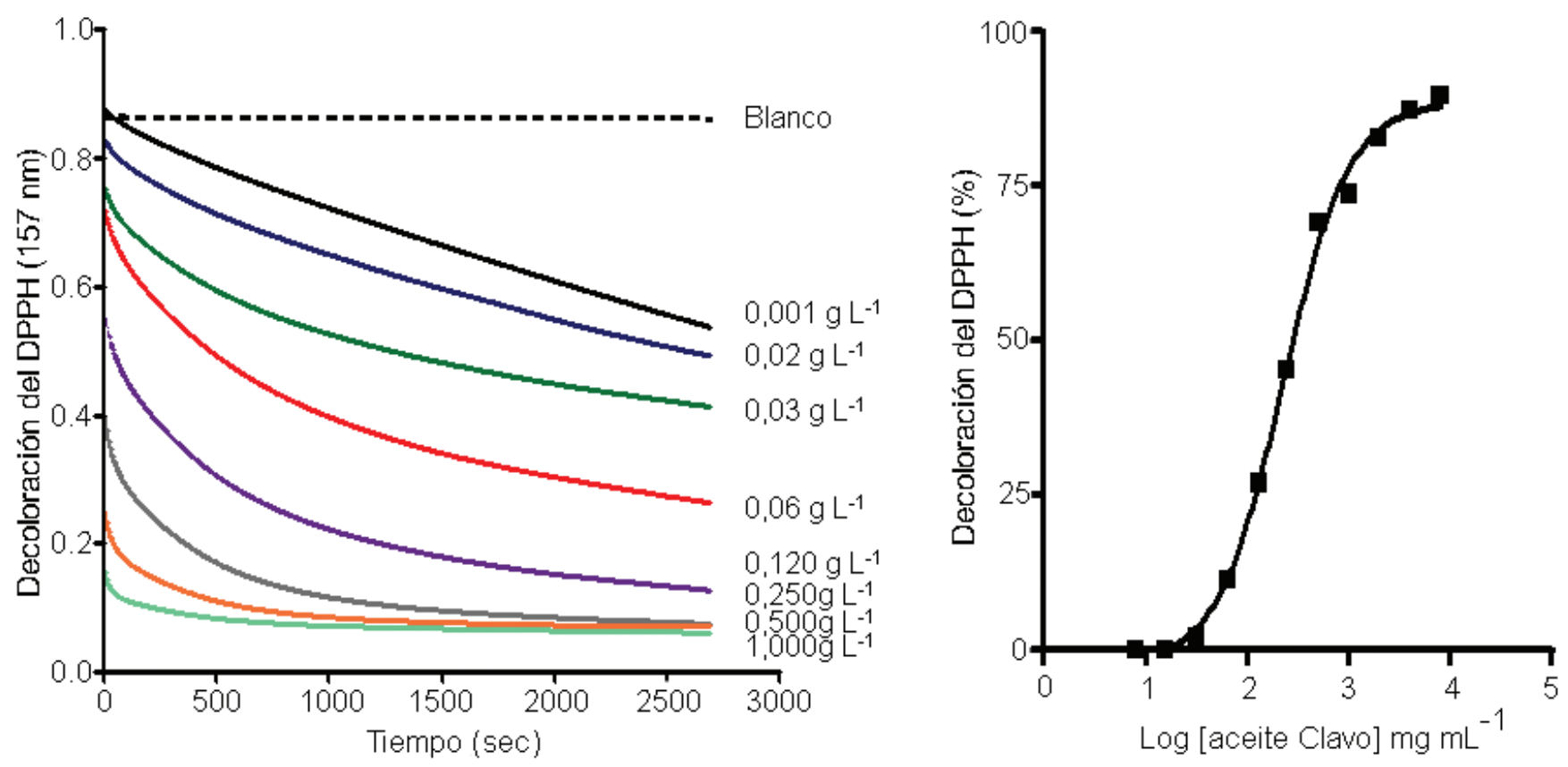

Figura 2S. A) Esquema del sistema para detección de antioxidantes volátiles emitidos desde fuentes vegetales. Bombona de nitrógeno 1; regulador de presión y flujo de nitrógeno 2; cámara de muestra 3; línea de conexión con el reactor 4; flujómetro 5; base del reactor 6; cámara de mezclado 7; cámara perforada en espiral para segunda fase de mezclado 8; cámara de muestreo 9; tubo de ventilación 10; bomba de doble pistón HPLC 11; orificio para muestreo de radical DPPH 12; orificio de retorno del radical DPPH desde el espectrofotómetro 13; espectrofotómetro de doble haz 14; PC con software para colección de los datos. B) Izquierda: detalle de la segunda cámara de mezclado indicada en A8. Orificio de entrada del radical en mezcla con gas de arrastre (1 mm d.i.) 1; orificio de salida del radical en mezcla con gas de arrastre (1 $\mathrm{mm}$ d.i.) 2; orificios de mezclado (d.i. $3 \mathrm{~mm}$ ) 3. Derecha: esquema detallado de las cámaras de mezclado y muestreo. Línea de entrada 4; orificio para retorno del radical 5; canal de conexión de la primera cámara de mezclado con la segunda 6; orificio para conexión del tubo de ventilación del sistema 7; orificio para muestreo del radical 